\title{
Research on Measures to Improve the Service Quality and Cultural Construction of University Canteens
}

\author{
Jialei Mao, Yanfei Shen, Li Wei, Xiaolan Mao, Junxue Li \\ Zhejiang Yuexiu University, Shaoxing, China \\ Email: 1305407913@qq.com
}

How to cite this paper: Mao, J.L., Shen, Y.F., Wei, L., Mao, X.L. and Li, J.X. (2022) Research on Measures to Improve the Service Quality and Cultural Construction of University Canteens. Open Access Library Journal, 9: e8401.

https://doi.org/10.4236/oalib.1108401

Received: January 26, 2022

Accepted: February 13, 2022

Published: February 16, 2022

Copyright $\odot 2022$ by author(s) and Open Access Library Inc.

This work is licensed under the Creative Commons Attribution International License (CC BY 4.0).

http://creativecommons.org/licenses/by/4.0/

\begin{abstract}
The Construction of Nutrition and Health Guidelines for Schools issued by National Health Commission of the People's Republic of China stressed the necessity to standardize the health and safety regulations of school canteens. As the primary venue of school logistic work, the canteen guarantees the health and safety of teachers' and students' diet. Furthermore, as an important part of school culture, canteen culture promotes personal growth and development, and enriches the connotation of school culture. Based on the investigation of the university canteen, this research finds out the existing problems, and proposes a series of measures to improve the service quality and cultural construction.
\end{abstract}

\section{Subject Areas}

Education Administration

\section{Keywords}

University Canteen, Service Quality, Cultural Construction

\section{1. 引言}

高校食堂作为学校后勤工作的中枢机关, 保证着学生饮食健康与安全。 近几年来, 校园食堂安全问题却层出不穷。河南新乡市封丘县赵岗镇戚城中 学 30 多名学生吃过 “营养午餐” 后, 疑似食物中毒; “华中农大” 食堂饭菜 价格不合理，一份梅菜扣肉 125 元; 武汉科技大学的南园餐厅，一年倒掉的 饭菜价值达上百万元。类似的新闻屡见不鲜, 从中我们可以看出食堂菜品质 量得不到保证、菜品定价过高、食物浪费现象等问题仍旧存在。并且餐饮外 
卖行业的涌起, 对高校食堂产生了巨大的冲击, 对菜品准备供应量、食堂人 流量都产生一定影响。同时, 高校食堂作为校园文化传播的平台, 发挥着立 德树人的重要作用。让学生在食堂既能感受到传统文化精神的熏陶, 又能接 受新时代新思想的洗礼。让食堂成为 “有温度” 的食堂, 加强对学生的思想 教育, 着力打造富有文化气息的就餐环境。因此, 对于如何提高校园食堂的 服务质量、加强文化的建设引发了我们的思考。

国内食堂大多采取自营或外包模式。所谓外包，即将食堂原材料的购买、 菜品的制作、售卖、卫生清洁等工作全部交由校外企业或个人承办。这种高 效便捷的模式，在一定程度上无法很好满足学生对不同菜品口味的需求，同 时无法达成统一合理的定价，在后厨制作卫生等方面也不能得到有效的监管。 而国外食堂大多采取学校自主监管模式。他们将满足学生的个性化需求作为 第一任务, 而将利润放在次位。他们会听取学生的建议及时修改菜单, 以便 更好地服务于学生。并且从源头监管食物的来源, 保障菜品的质量。这种模 式, 使食堂得到最有效的管理, 使学生的利益达到最大化。国内国外对于校 园食堂管理的模式与方法都有所不同，但从中可以互相借鉴，取长补短。国 内校园食堂可以汲取国外校园食堂管理的成功经验, 进而更好地满足学生的 需求。

\section{2. 校园食堂餐饮现状及数据分析}

校园食堂的安全与卫生不仅关系全体师生的身体健康与生命安全，更关 系着校园内部的和谐与稳定。现以浙江越秀外国语学院为例, 通过校园食堂 的实地调查，对其现状及数据进行分析。

\section{1. 人流量}

根据研究人员在不同时间点对我校食堂就餐人数的统计, 得出结论为: 第一，在饭点期间就餐人数达到高峰。如表 1 显示，研究人员在 10 月 12 日

Table 1. Statistics of the number of diners in the canteen

表 1. 食堂就餐人数统计数据

\begin{tabular}{cccc}
\hline 日期 & 时间 & 人数 & 合计 \\
\hline 10.12 & $11: 40 \sim 12: 00$ & 274 & 473 \\
10.12 & $17: 00 \sim 17: 20$ & 199 & \\
10.13 & $11: 40 \sim 12: 00$ & 178 & 405 \\
10.13 & $17: 00 \sim 17: 20$ & 227 & \\
10.14 & $11: 40 \sim 12: 00$ & 213 & 409 \\
10.14 & $17: 00 \sim 17: 20$ & 196 & 437 \\
10.15 & $11: 40 \sim 12: 00$ & 208 & \\
10.15 & $17: 00 \sim 17: 20$ & 229 & 420 \\
10.16 & $11: 40 \sim 12: 00$ & 214 & \\
10.16 & $17: 00 \sim 17: 20$ & 206 & \\
\hline
\end{tabular}


至 10 月 16 日连续五天中的饭点期间, 即上下午下课 20 分钟内获取到的食堂 就餐人数高峰值。第二, 各地特色小吃窗口就餐人数占比较大。调查数据显 示, 在就餐高峰时期, 特色小吃窗口人数均在 $25 \sim 45$ 人。由于部分班级下课 时间点相同, 容易造成食堂饭点时刻就餐人数偏多现象, 进而导致点餐和取 餐的队伍混在一起, 没有清晰的界限划分, 导致秩序混乱、碰撞事件频发。

\section{2. 食堂设备}

就我校而言, 食堂目前仅有二楼装有监控显示器, 并没有覆盖至其他楼 层。每层楼都有餐盘传送带, 但传送带的灵敏度不高, 不能及时回收和清理 餐盘。并且周一至周五时段的早读(7:20 开始)导致前半个小时二楼就餐人数较 多，而传送带在这个时间段并不工作，导致餐盘堆积过多，严重影响食堂整 洁干净的面貌, 如图 1 所示。我校食堂设备的覆盖面不广、工作效率不高, 因此导致相关问题的产生。
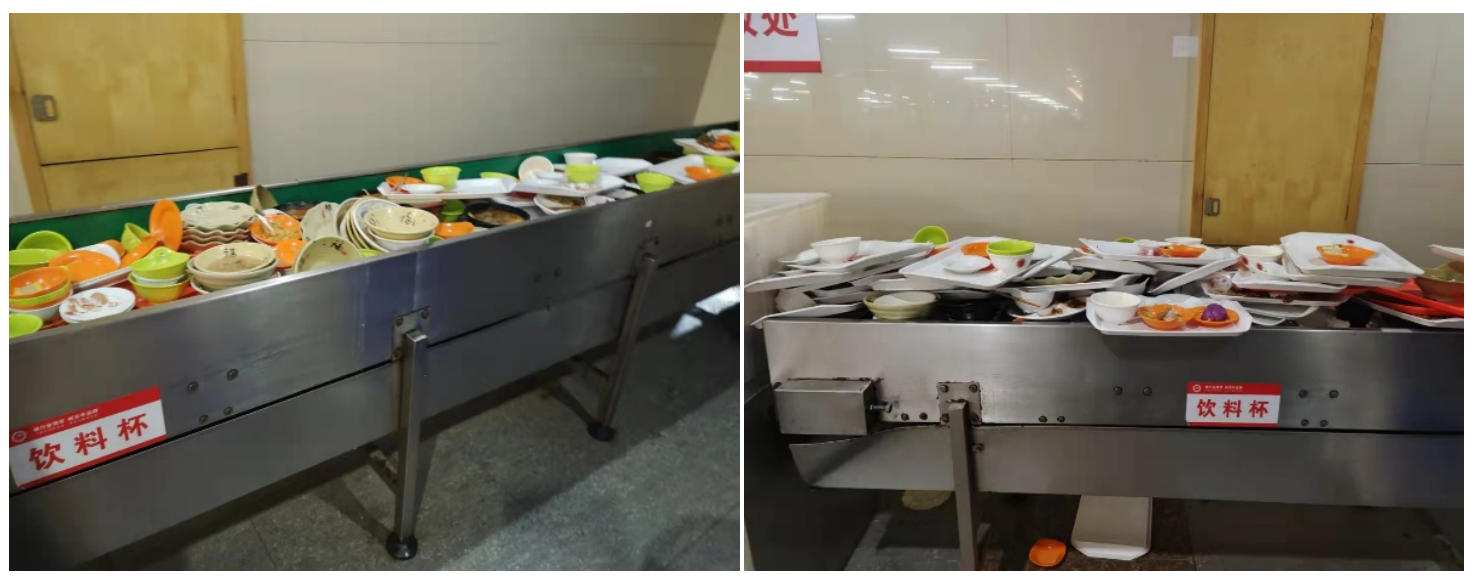

Figure 1. Trays piling up on conveyor belts during breakfast

图 1. 早餐时段传送带餐盘堆积现象图

\section{3. 菜品质量与价格}

食堂能够满足学生一日三餐, 并且营业时间相对较长, 能满足学生不同 时间段的需求。在食堂菜品方面, 种类较为丰富, 主要以江浙沪地区为主, 虽能够满足大部分学生口味, 但口味相对统一, 江浙沪以外地区的口味覆盖 面较少。菜品较为固定, 更换调整新菜品的次数不多。价格方面较其他学校 偏贵, 但与校外价格相比较便宜。有学生反映偶尔从食堂菜品中发现异物, 结合实际情况, 研究人员认为造成这种情况的主要原因有以下几点: 一是食 堂菜品属于大批量进货, 质量难以得到很好的保证; 二是食堂工作人员清洗 处理工作不够细致; 三是㕑房内部环境卫生安全未做到位。综上所述, 我校 食堂菜品种类多, 价格适中, 但餐品质量仍有进步空间。

\section{4. 学生满意度}

为获取我校学生对食堂各方面的满意情况, 项目成员通过问卷调查的形 式, 收集数据。如表 2 数据, 可以看出, 我校学生对食堂一楼餐品价格满意 
度较低, 其余均在平均水平左右。相较于食堂一楼, 我校学生对食堂二楼餐 厅菜品价格、品质以及卫生的满意度较低, 其余均在平均水平左右。对三楼 小吃档口而言, 我校学生对食品卫生和菜品新鲜度以及种类的关注度较高。 总体来说, 我校学生对食堂满意度较低, 食堂各方面反映出的问题有待解决。

Table 2. Survey data of students' satisfaction with canteen (10-point scale)

表 2. 学生对食堂满意度调查数据(10 分制)

\begin{tabular}{ccc}
\hline 调查内容 & 平均分(一楼) & 平均分(二楼) \\
\hline 明码标价及打卡准确率 & 6.97 & 6.56 \\
菜肴品质 & 6.53 & 4.81 \\
菜品种类是够满足需求 & 5.82 & 5.09 \\
菜有价格 & 3.71 & 4.72 \\
餐具卫生 & 6.19 & 4.95 \\
就餐环境 & 7.76 & 6.98 \\
工作人员服务态度 & 8.16 & 8.25 \\
保温措施 & 8.03 & 7.08 \\
整体满意度 & 6.19 & 5.47 \\
\hline
\end{tabular}

\section{3. 校园食堂餐饮存在的问题}

基于对校园食堂现状与数据分析, 我校食堂还有较多待完善的地方, 其 中体现的问题主要为以下三类。

\section{1. 食堂卫生问题}

根据我校食堂各个楼层的问卷调查结果显示, 学生对食堂卫生情况评价 一般。经过对食堂深度观察，发现由于传送带运作不及时，导致就餐时间段 餐盘堆积，堆积时间过久进而造成传送带区域散发异味; 由于食堂工作人员 清理污渍不彻底，导致地面粘喴; 由于垃圾清理不及时，导致㴻余垃圾散发 异味。此类情况严重影响食堂卫生面貌, 影响学生就餐心情。综上, 食堂卫 生成为亟待解决的问题。

\section{2. 食堂监管问题}

我校食堂大部分窗口餐品于后厨制作，学生难以看到制作过程，这促使 学生对食堂餐品的信任度降低。我校食堂虽然有监控显示屏, 但仅覆盖二楼 部分窗口, 且餐品制作过程可见性低。因此, 为增加学生信任度, 保证后厨 制作的干净卫生，我校应加强食堂监管。

\section{3. 食堂浪费问题}

通过调查发现食堂存在浪费现象, 因为菜品口味不适合、菜量过大等主 观或客观原因，导致餐盘中有大量剩菜剩饭剩余。而后厨人员将剩饭剩菜全 部倒置于一个桶内, 无法很好的进行垃圾分类。同时经观察多数学生还会选 
择打包餐品, 不选择在食堂就餐, 也会造成塑料袋、打包盒、一次性餐具等 白色污染的产生。因此如何减少浪费现象成为我校食堂亟待解决的问题。

\section{4. 校园食堂服务质量与文化建设提升的具体措施}

\section{1. 提升食堂服务质量}

\subsection{1. “减脂轻食餐”}

身材管理已是当下的热门话题。很多年轻人不仅通过健身来控制身材, 也通过吃低热量、低脂肪的食物来补充营养。为吸引更多学生去食堂就餐, 特此推出 “减脂轻食餐” 。这一举措既符合当下的潮流趋势, 也满足学生关 于身材管理的心理需求。可以根据人体需要的营养搭配不同类型、不同价格 的营养餐, 根据成年人的膳食摄入标准去搭配轻食餐。其中食物要多样化, 以谷类为主, 蔬菜水果充足。轻食餐要清淡, 少盐, 蛋白质摄入以牛奶、鸡 蛋为主 $[1]$ 。

推出 “减脂轻食餐” 有利于提升食堂餐饮质量, 保证了菜品的丰富多样, 也均衡搭配各类营养要素, 弥补食堂菜品单一的劣势。让更多学生减少点外 卖的不良现象, 促进学生的健康发展。同时为大学食堂的餐饮服务提供了一 种创新模式, 进一步提升食堂的餐饮质量。

\subsection{2. “明欴亮灶”}

经调查, 多家商家店铺的餐饮制作过程是半透明化, 未能全面的向学生 公开后厨制作的全过程。并且多名学生反映在食堂吃出虫子、钢丝球等不洁 物, 这说明学校食堂的餐饮卫生无法得到有力保障。因此改善学校食堂的卫 生问题是重中之重, 应当实现完全透明化的明厨亮灶, 让学生实时查看学校 厨房卫生、后厨制作等流程探索学校食品安全监管的新模式, 营造学校食品 安全的格局。

明厨亮灶的实施方式主要包括 “透明厨房” “视频厨房” 以及 “ “互联 网+” 明厨亮灶”。“透明厨房” 是指后㕑建造透明玻璃窗、透明玻璃幕墙, 让学生能够直观的看到后厨制作餐饮的流程, 实现公开透明化。“视频厨房” 指在餐饮制作场合安装摄像设备, 并通过展示屏看到加工制作餐饮的全流程。

““互联网+”明厨亮灶” 指在餐饮制作场合安装摄像设备, 通过线上方式可 以看到餐饮制作过程、食品溯源信息、监督公示以及提供和消费者满意度测 评的方式[2]。通过大数据对人员健康、地面卫生等情况进行实时监查, 将隐 患第一时间排除。

明厨亮灶能够让学生实地查看后厨的制作流程, 这有利于改善学校食堂 的卫生问题, 逐步解决学校食堂的食品安全问题, 提升食堂口碑。同时加强 了食堂餐饮制作人员的自我监督责任感, 学生的就餐卫生问题也能得到保障。

\subsection{3. “一左一右队伍线”}

学校食堂在地上设置了分隔线, 也在每家商铺的两边拉上了隔离带, 但 未能在学生排队等餐时充分利用起来。由于吃饭时间人流量大, 许多学生在 排队点餐、等餐时秩序混乱, 导致学生等待时间过长、打包盒拿错等现象频 繁出现。为了减少学生的等待时间, 提升商家的服务效率, 故设置 “一左一 
右队伍线”。在左右两侧划分明显的队伍线, 开始设置队伍线时配备工作人 员引导学生一左一右排队, 分散人流到不同的窗口去点餐, 逐步养成学生自 觉按队伍线排队的习惯, 让学生有序的进行食堂就餐。

设置 “一左一右队伍线” 能够让学生在点餐、等餐时有序排队, 减少学 生的等待时间。商家能够快速便捷的分清点餐、等餐的学生, 方便商家给学 生拿对打包餐食, 降低错误率, 在保证速度的同时也能提升效率。

\subsection{4. “重力感应传送带”}

学校食堂在餐盘回收处建有传送带, 但仍有诸多弊端, 如在特定时间段 开启导致其他时间餐盘堆积, 影响食堂环境的整洁度。回收餐盘仍然需要人 力, 耗费大量时间。为提升学校回收餐盘的效率, 节省人力和物力, 节约资 源。因此建立重力感应传送带帮助食堂工作人员回收餐具, 做好食堂清洁工 作。找专门的技术人员安装重力感应传送带, 餐盘一放上去传送带就能感知 到。并且能够及时感应到餐盘放置时间, 传送带会自动启动, 将餐盘送到后 厛，方便食堂工作人员清洗餐盘。

重力感应传送带是高校食堂的一种创新举措, 致力于解决食堂餐盘回收 的问题, 便于提升食堂的清洁度和美观性, 给学校食堂工作人员提供了便利, 能够在回收餐具上节省时间, 提高清洁效率。

\section{2. 建设食堂文化}

\subsection{1. “考试助力套餐”}

为助力四六级、研究生入学等大型考试, 在考试周期间推出一系列有关 考试谐音套餐。为实行此计划, 研究人员可以于四六级考试前同食堂工作人 员进行食谱沟通, 结合实际情况最后将菜单定为: 回锅肉 + 竹笋, 寓意为 “胸 有成竹” ; 甜椒炒肉+西红柿炒鸡蛋, 寓意为 “红红火火” ; 宫保鸡丁 + 玉 米粒, 寓意为 “大吉大利”; 蒸蛋 + 土豆炒肉, 寓意为 “蒸蒸日上” 等套餐, 并在考试前夕在食堂正式推出。

“考试助力套餐” 不仅解决了排队拥挤问题, 还节约了考生的时间; 不 仅吸引了更多同学于食堂就餐, 还为将要考试的同学给予祝福, 增加食堂的 趣味性。在一定程度上让学生感受到食堂的温暖。

\subsection{2. “传统美食节日分享会”}

为引导学生弘扬和传承中华优秀传统文化, 在中国传统节日时举办有关 节日传统习俗的活动。参考各高校组办的关于 “传统美食节日” 此类主题的 活动, 项目成员与食堂工作人员及学生会进行沟通, 定于中秋节筹办 “传统 美食节日分享会”。结合实际情况以及菜品难易程度, 食堂将推出以下菜品:

“芋头”，寓意辟邪消灾，有表示不信邪之意; “南瓜糯米饭”，寓意生活 甜甜蜜蜜、和和美美; “藕盒子”，寓意团圆圆满之意。

同时与学生会对接, 提前发推送进行活动预热。活动开展于农历八月十 五, 通过对参加活动的同学进行分组, 各组抢答关于中秋节的传统文化知识 获得免费品尝食堂所准备的三种菜品的机会, 最终比较积分, 胜利的小组获 得月饼为奖品。 
通过举办 “传统美食节日分享会” 的活动, 将传统文化潜移默化在食堂 中传播, 让同学在轻松欢快的氛围中了解更多关于传统节日的知识, 起到弘 扬传统文化的作用。

\subsection{3. “众神归位”}

为保证食堂桌椅整齐摆放, 扩大行走空间, 提升舒适感以及整洁感, 与 食堂后勤部联合实行 “众神归位” 措施。除食堂固定桌椅外, 在每张可移动 桌椅处贴放或摆放 “众神归位” 字样 KT 板, 提醒同学离开座位时有效且及 时的归位桌椅, 不阻碍其他借过找位置的同学。

通过此次活动的实行, 食堂整体整洁情况得到改善, 桌椅摆放比之前更 加整齐, 桌间空隙加大进而使行走空间增大, 使食堂看起来更加开阔整洁。

\section{3. 开展各类比赛活动}

\subsection{1. “21 天光盘打卡行动” + “奖励机制”}

为呼吁学生积极堂食, 减少外带, 缓解白色污染现象。并且养成节约、 不浪费粮食的好习惯。项目成员与学生会组织可以联合开展 “ 21 天光盘打卡 行动” , 借此鼓励学生坚持光盘行动。活动前, 相关学生会发送推送提前预 热, 得知大致报名人数后讨论奖励机制。在与老师沟通后, 确定奖励机制为

“光盘打卡满 21 天的同学可获得荣誉奖状”。同时提前建立钉钉打卡群, 以 供参赛同学发布光盘照片, 完成打卡, 也方便结果统计。

“21 天光盘打卡行动” 在积极倡导勤俭文化、节约文化、绿色文化的同 时, 也培养了同学们珍惜粮食、保护资源环境的意识。

\subsection{2. “共享棏房”}

根据调查问卷结果显示, 有部分同学不习惯于食堂饭菜, 想有自己做饭 的机会。通过项目成员商讨, 提出 “共享厨房” 这一想法。据了解, 我校的 教官队曾经借用食堂器具开展过包饺子活动, 因此 “共享厨房” 这一想法有 机会得以实现。

通过与食堂工作人员协商, “共享厨房” 可以顺利开展。但由于实际情 况限制, 比如在固定用餐期间, 食堂后厨处于忙碌情况, 此时并不便于开设 “共享厨房” 等情况。所以对活动开展定有以下几条限制: 一、食堂忙碌期 间不予以开设 “共享厨房”，㕑房可使用时间具体见当天安排表(贴于门口); 二、为防止拥挤情况, 使用 “共享厨房” 需提前预约; 三、食材不予以提供, 器具不予以限制使用; 四、为不妨碍下一位同学及食堂工作人员使用厨房, 在使用 “共享厨房” 后需保持厨房整洁, 并将使用过的器具归回原位。

“共享厨房” 预约使用活动, 在学习厨艺和享受自己劳动成果的同时可 以加深对饮食服务工作的理解和对饮食文化的感悟, 增强学生对饮食服务工 作的认同感和对劳动者的敬重感。

\subsection{3. “拼一拼”}

为吸引较多同学于食堂就餐, 在食堂原有基础上可以开展拼饭活动。在 固定窗口就餐, 两人九折, 三人八折, 三人以上七折。并且购买饭菜达到一 
定价格后, 可根据就餐人数赠送饮料。活动开始前于公众号内发布推送进行 活动预热, 并标明转发此推送可于该窗口进行抽奖。通过抽奖有机会获得不 定额优惠券，购餐价格达到规定额度后便可用于抵消。

通过在一些食堂窗口开设 “拼一拼” 活动, 使得同学组队吃饭可以享受 一定的优惠折扣。在一定程度上, 吸引学生就餐, 促进同学之间的相互联系 与交流，同时推动食堂部分窗口更好地运行。

\section{4. 加强推广宣传}

\subsection{1. 创建并推广“多滋多味小食堂” 公众号}

通过网络这一传播媒介让更多学生了解食堂, 增加对食堂的关注度, 扩 大其影响力。通过在公众号平台创建 “多滋多味小食堂” 公众号, 并定期在 上面发布食堂近期特色菜品、优惠福利、活动预告等推送, 吸引学生的注意 力，鼓励积极去食堂就餐。

在 “互联网+” 时代背景下, 将线上与线下结合, 通过网络的即时性弥补 线下宣传的局限性, 开创食堂宣传的新模式。通过加大宣传力度, 激发学生 对食堂餐品的兴趣, 进而积极推动食堂的发展。

\subsection{2. 与后勤保障处紧密合作}

后勤服务保障处是为给广大师生提供优质服务而存在的。保障处设有专 门的工作人员负责和食堂经营者对接, 做好食堂管理工作。保障处可以结合 学生需求, 改进学校食堂在服务和管理方面的不足之处, 进而满足来自不同 地域、不同民族的学生的口味, 提升学生对食堂菜品的满意度。

针对学校食堂存在的一些问题, 结合学校的实际情况, 提出以下对策:

1) 密切关注后勤保障处关于食堂的管理运行流程, 重点关注食堂的饭菜 价格制定、饭菜质量把关等过程。

2) 秉承 “以学生为本” 的理念, 增强食堂工作人员为学生服务的自觉性, 在把握学生食堂饭菜质量的同时，根据学生的不同口味需要提供丰富多样的 菜品。

3）让学生也广泛参与到食堂的后勤管理中来, 发现问题可以向食堂管理 人员及时反馈，提出改进意见[3]。

4) 不定时展开调查, 关注学生对食堂菜品种类、质量、卫生等问题的满意 度, 持续改进, 组织各类丰富多样的活动, 让学生参与食堂餐品的制作过程。

5) 对学校食堂工作人员展开培训, 提升食堂工作人员的思想道德水平, 可 以对食堂工作人员提供食品营养方面的知识, 为学生提供营养成分充足的菜品。

通过在理论方面指导高校的食堂管理，推动着后勤服务的健康有序发展， 有利于因地制宜解决学校食堂管理存在的一些现实问题。并且充分了解师生 的饮食需求和期望, 详细分析高校食堂存在的问题, 进而提出详细有针对性 的解决方法。

\section{5. 措施实践的意义}

在当前学生学业压力、生活压力不断增加的情况下, 对于生活质量的要 
求也逐步提高, 因而学校食堂相关情况受到更为广泛的关注。我们依托现有 的关于食堂餐饮调查数据, 提出一系列关于改善校园餐饮服务的建议与措施。 首先，我们将采用现阶段较为流行的生活方式，倡导校园绿色健康饮食; 其 次，我们将饮食文化与校园食堂相结合，以宣扬中华民族传统文化，提升学 生民族文化的认同感; 最后，我们将采用新颖的宣传推广方式，建立专属公 众号, 举办各类活动比赛, 鼓励学生食堂就餐, 减少外带、外卖现象。通过 线上与线下的互动联系，力求对高校食堂餐饮的问题进行改善，提升餐饮服 务质量, 让学生能注重健康饮食生活方式的同时, 也能提升自我的素质能力。 进而实现校园餐饮服务到 “胃”，餐饮文化津津有味。

\section{6. 结语}

高校食堂作为学校后勤服务工作的中枢，保证着师生日常饮食的健康安 全。本文从高校食堂服务质量与文化建设两个角度, 通过采取一系列创新性 的举措：推出减脂轻食餐、改变排队路线、将食堂文化与传统文化相结合、 开展一系列奖励机制的活动、推出热门应用等, 以期更好满足学生的各类需 求，进而达到提升餐饮服务品质、发挥文化熏陶等作用。在保护师生 “舌尖” 安全的同时, 加强树立食堂形象, 让食堂成为有 “温度” 的食堂, 打响学校 的金字招牌。

然而, 高校食堂的创新与改造, 需要师生、学校、社会的共同努力。让 食堂不仅是食堂, 更能成为学堂, 让学生在食堂中能有所学、有所悟、有所 感, 努力践行新时代新青年精神。

\section{基金项目}

2021 年国家级大学生创新创业训练计划项目 “食堂服务处处到 “胃” 食 堂文化焕然一新” (项目编号：202112792015); 浙江省高等学校课程思政教学 研究项目 “一流课程建设背景下大学英语课程思政内涵和实施路径探究” ; 2021 年度校级 “课程思政” ( “三进”专项)示范课程《综合英语》。

\section{Conflicts of Interest}

The authors declare no conflicts of interest.

\section{References}

[1] 刘志友, 姬金辰. 大连市高校食堂营养餐的开发对策研究 [J]. 现代商贸工业, 2017(19): 79-80. https://doi.org/10.19311/j.cnki.1672-3198.2017.19.038

[2] 戴婵. 食品安全监管方式创新研究 [D]: [硕士学位论文]. 上海: 上海交通大学, 2019. https://doi.org/10.27307/d.cnki.gsjtu.2019.001081

[3] 陈研. 后勤服务社会化背景下高校学生食堂管理研究[D]: [硕士学位论文]. 福州: 福建农林大学, 2014. 


\section{Appendix (Abstract and Keywords in Chinese)}

\section{高校食堂服务质量与文化建设提升措施研究}

摘要：国家卫生健康委印发的《营养与健康学校建设指南》中强调指出需要 规范校园食堂卫生安全。食堂作为学校后勤工作的主要场所, 保证着师生饮 食的健康与安全。同时, 食堂文化作为校园文化的重要组成部分, 推动着个 人的成长与发展，丰富着校园文化的内涵。本研究在对高校食堂进行实地调 查的基础上，发现其存在的问题，并提出一系列关于提升餐饮服务质量与食 堂文化建设的措施。

关键词：高校食堂，服务质量，文化建设 